\title{
Sharp bounds for Sándor mean in terms of arithmetic, geometric and harmonic means
}

Wei-Mao Qian', Yu-Ming Chu ${ }^{*}$ and Xiao-Hui Zhang ${ }^{2}$

${ }^{*}$ Correspondence:

chuyuming2005@126.com

${ }^{2}$ School of Mathematics and Computation Sciences, Hunan City University, Yiyang, 413000, China Full list of author information is available at the end of the article

$$
\begin{aligned}
& \text { Abstract } \\
& \text { In the article, we present the best possible parameters } \alpha_{1}, \alpha_{2}, \beta_{1}, \beta_{2} \in(0,1) \text { and } \\
& \alpha_{3,} \alpha_{4}, \beta_{3}, \beta_{4} \in(0,1 / 2) \text { such that the double inequalities } \\
& \qquad \begin{array}{c}
\alpha_{1} A(a, b)+\left(1-\alpha_{1}\right) H(a, b)<X(a, b)<\beta_{1} A(a, b)+\left(1-\beta_{1}\right) H(a, b) \text {, } \\
\alpha_{2} A(a, b)+\left(1-\alpha_{2}\right) G(a, b)<X(a, b)<\beta_{2} A(a, b)+\left(1-\beta_{2}\right) G(a, b), \\
H\left[\alpha_{3} a+\left(1-\alpha_{3}\right) b, \alpha_{3} b+\left(1-\alpha_{3}\right) a\right]<X(a, b)<H\left[\beta_{3} a+\left(1-\beta_{3}\right) b, \beta_{3} b+\left(1-\beta_{3}\right) a\right] \\
\quad G\left[\alpha_{4} a+\left(1-\alpha_{4}\right) b, \alpha_{4} b+\left(1-\alpha_{4}\right) a\right]<X(a, b)<G\left[\beta_{4} a+\left(1-\beta_{4}\right) b, \beta_{4} b+\left(1-\beta_{4}\right) a\right]
\end{array}
\end{aligned}
$$

hold for all $a, b>0$ with $a \neq b$. Here, $X(a, b), A(a, b), G(a, b)$ and $H(a, b)$ are the Sándor, arithmetic, geometric and harmonic means of $a$ and $b$, respectively.

MSC: $26 \mathrm{E} 60$

Keywords: Sándor mean; arithmetic mean; geometric mean; harmonic mean

\section{Introduction}

Let $r \in \mathbb{R}$ and $a, b>0$ with $a \neq b$. Then the harmonic mean $H(a, b)$, geometric mean $G(a, b)$, logarithmic mean $L(a, b)$, Seiffert mean $P(a, b)$, arithmetic mean $A(a, b)$, Sándor mean $X(a, b)[1]$ and $r$ th power mean $M_{r}(a, b)$ of $a$ and $b$ are, respectively, defined by

$$
\begin{aligned}
& H(a, b)=\frac{2 a b}{a+b}, \quad G(a, b)=\sqrt{a b}, \quad L(a, b)=\frac{a-b}{\log a-\log b}, \\
& P(a, b)=\frac{a-b}{2 \arcsin \left(\frac{a-b}{a+b}\right)}, \quad A(a, b)=\frac{a+b}{2}, \quad X(a, b)=A(a, b) e^{\frac{G(a, b)}{P(a, b)}-1}
\end{aligned}
$$

and

$$
M_{r}(a, b)=\left(\frac{a^{r}+b^{r}}{2}\right)^{1 / r} \quad(r \neq 0), \quad M_{0}(a, b)=\sqrt{a b} .
$$

It is well known that $M_{r}(a, b)$ is continuous and strictly increasing with respect to $r \in \mathbb{R}$ for fixed $a, b>0$ with $a \neq b$, and the inequalities

$$
H(a, b)<G(a, b)<L(a, b)<P(a, b)<A(a, b)
$$

hold for all $a, b>0$ with $a \neq b$.

(c) 2015 Qian et al. This article is distributed under the terms of the Creative Commons Attribution 4.0 International License (http://creativecommons.org/licenses/by/4.0/), which permits unrestricted use, distribution, and reproduction in any medium, provided you give appropriate credit to the original author(s) and the source, provide a link to the Creative Commons license, and indicate if changes were made. 
Recently, the Sándor mean has attracted the attention of several researchers. In [2], Sándor established the inequalities

$$
\begin{aligned}
& X(a, b)<\frac{P^{2}(a, b)}{A(a, b)}, \quad \frac{A(a, b) G(a, b)}{P(a, b)}<X(a, b)<\frac{A(a, b) P(a, b)}{2 P(a, b)-G(a, b)} \\
& X(a, b)>\frac{A(a, b) L(a, b)}{P(a, b)} e^{\frac{G(a, b)}{L(a, b)}-1}, \quad X(a, b)>\frac{A(a, b)[P(a, b)+G(a, b)]}{3 P(a, b)-G(a, b)}, \\
& \frac{A^{2}(a, b) G(a, b)}{P(a, b) L(a, b)} e^{\frac{L(a, b)}{A(a, b)}-1}<X(a, b)<A(a, b)\left[\frac{1}{e}+\left(1-\frac{1}{e}\right) \frac{G(a, b)}{P(a, b)}\right] \\
& A(a, b)+G(a, b)-P(a, b)<X(a, b)<A^{-1 / 3}(a, b)\left[\frac{A(a, b)+G(a, b)}{2}\right]^{4 / 3}, \\
& P^{1 /(\log \pi-\log 2)(a, b) A^{1-1 /(\log \pi-\log 2)}(a, b)} \\
& \quad<X(a, b)<P^{-1}(a, b)\left[\frac{A(a, b)+G(a, b)}{2}\right]^{2}
\end{aligned}
$$

for all $a, b>0$ with $a \neq b$.

Yang et al. [3] proved that the double inequality

$$
M_{p}(a, b)<X(a, b)<M_{q}(a, b)
$$

holds for all $a, b>0$ with $a \neq b$ if and only if $p \leq 1 / 3$ and $q \geq \log 2 /(1+\log 2)=0.4903 \ldots$ In [4], Zhou et al. proved that the double inequality

$$
H_{\alpha}(a, b)<X(a, b)<H_{\beta}(a, b)
$$

holds for all $a, b>0$ with $a \neq b$ if and only if $\alpha \leq 1 / 2$ and $\beta \geq \log 3 /(1+\log 2)=0.6488 \ldots$, where $H_{p}(a, b)=\left[\left(a^{p}+(a b)^{p / 2}+b^{p}\right) / 3\right]^{1 / p}(p \neq 0)$ and $H_{0}(p)=\sqrt{a b}$ is the $p$ th power-type Heronian mean of $a$ and $b$.

Inequalities (1.4) and (1.5) together with the identities $H(a, b)=M_{-1}(a, b), G(a, b)=$ $M_{0}(a, b)$ and $A(a, b)=M_{1}(a, b)$ lead to the inequalities

$$
H(a, b)<G(a, b)<X(a, b)<A(a, b)
$$

for all $a, b>0$ with $a \neq b$.

Let $a, b>0$ with $a \neq b, x \in[0,1 / 2], f(x)=H[x a+(1-x) b, x b+(1-x) a]$ and $g(x)=G[x a+$ $(1-x) b, x b+(1-x) a]$. Then both functions $f$ and $g$ are continuous and strictly increasing on $[0,1 / 2]$. Note that

$$
f(0)=H(a, b)<X(a, b)<f(1 / 2)=A(a, b)
$$

and

$$
g(0)=G(a, b)<X(a, b)<g(1 / 2)=A(a, b) .
$$


Motivated by inequalities (1.7)-(1.9), we naturally ask: what are the best possible parameters $\alpha_{1}, \alpha_{2}, \beta_{1}, \beta_{2} \in(0,1)$ and $\alpha_{3}, \alpha_{4}, \beta_{3}, \beta_{4} \in(0,1 / 2)$ such that the double inequalities

$$
\begin{aligned}
& \alpha_{1} A(a, b)+\left(1-\alpha_{1}\right) H(a, b)<X(a, b)<\beta_{1} A(a, b)+\left(1-\beta_{1}\right) H(a, b), \\
& \alpha_{2} A(a, b)+\left(1-\alpha_{2}\right) G(a, b)<X(a, b)<\beta_{2} A(a, b)+\left(1-\beta_{2}\right) G(a, b), \\
& H\left[\alpha_{3} a+\left(1-\alpha_{3}\right) b, \alpha_{3} b+\left(1-\alpha_{3}\right) a\right]<X(a, b)<H\left[\beta_{3} a+\left(1-\beta_{3}\right) b, \beta_{3} b+\left(1-\beta_{3}\right) a\right], \\
& G\left[\alpha_{4} a+\left(1-\alpha_{4}\right) b, \alpha_{4} b+\left(1-\alpha_{4}\right) a\right]<X(a, b)<G\left[\beta_{4} a+\left(1-\beta_{4}\right) b, \beta_{4} b+\left(1-\beta_{4}\right) a\right]
\end{aligned}
$$

hold for all $a, b>0$ with $a \neq b$ ? The purpose of this paper is to answer this question.

\section{Lemmas}

In order to prove our main results, we need four lemmas, which we present in this section.

Lemma 2.1 Let $p \in(0,1)$ and

$$
f(x)=\frac{x \sqrt{1-x^{2}}\left[(1-p) x^{2}+1\right]}{p+(1-p)\left(1-x^{2}\right)}-\arcsin (x) .
$$

Then the following statements are true:

(1) If $p=2 / 3$, then $f(x)<0$ for all $x \in(0,1)$.

(2) If $p=1 / e$, then there exists $\lambda_{1} \in(0,1)$ such that $f(x)>0$ for $x \in\left(0, \lambda_{1}\right)$ and $f(x)<0$ for $x \in\left(\lambda_{1}, 1\right)$.

Proof Simple computations lead to

$$
\begin{aligned}
& f(0)=0, \quad f(1)=-\frac{\pi}{2} \\
& f^{\prime}(x)=\frac{2 x^{2}}{\sqrt{1-x^{2}}\left[p+(1-p)\left(1-x^{2}\right)\right]^{2}} f_{1}(x),
\end{aligned}
$$

where

$$
f_{1}(x)=(1-p)^{2} x^{4}-(1-p)(3-p) x^{2}+2-3 p .
$$

(1) If $p=2 / 3$, then (2.4) leads to

$$
f_{1}(x)=-\frac{x^{2}}{9}\left(7-x^{2}\right)<0
$$

for $x \in(0,1)$.

Therefore, $f(x)<0$ for $x \in(0,1)$ follows easily from (2.2), (2.3) and (2.5).

(2) If $p=1 / e$, then (2.4) leads to

$$
\begin{aligned}
& f_{1}(0)=\frac{2 e-3}{e}>0, \quad f_{1}(1)=-\frac{1}{e}<0, \\
& f_{1}^{\prime}(x)=2(1-p)\left[2(1-p) x^{2}-(3-p)\right] x<-2\left(1-p^{2}\right) x<0
\end{aligned}
$$

for $x \in(0,1)$. 
From (2.6) and (2.7) we clearly see that there exists $\lambda_{0} \in(0,1)$ such that $f_{1}(x)>0$ for $x \in\left(0, \lambda_{0}\right)$ and $f_{1}(x)<0$ for $x \in\left(\lambda_{0}, 1\right)$.

We divide the proof into two cases.

Case 1. $x \in\left(0, \lambda_{0}\right]$. Then $f(x)>0$ follows easily from (2.2) and (2.3) together with $f_{1}(x)>0$ on the interval $\left(0, \lambda_{0}\right)$.

Case 2. $x \in\left(\lambda_{0}, 1\right)$. Then $(2.3)$ and $f_{1}(x)<0$ on the interval $\left(\lambda_{0}, 1\right)$ lead to the conclusion that $f(x)$ is strictly decreasing on $\left[\lambda_{0}, 1\right)$.

From (2.2) and $f\left(\lambda_{0}\right)>0$ together with the monotonicity of $f(x)$ on $\left[\lambda_{0}, 1\right)$ we clearly see that there exists $\lambda_{1} \in\left(\lambda_{0}, 1\right) \subset(0,1)$ such that $f(x)>0$ for $x \in\left(\lambda_{0}, \lambda_{1}\right)$ and $f(x)<0$ for $x \in\left(\lambda_{1}, 1\right)$.

Lemma 2.2 Let $p \in(0,1)$ and

$$
g(x)=\frac{p x \sqrt{1-x^{2}}+(1-p) x}{(1-p) \sqrt{1-x^{2}}+p}-\arcsin (x) .
$$

\section{Then the following statements are true:}

(1) If $p=1 / 3$, then $g(x)>0$ for all $x \in(0,1)$.

(2) If $p=1 / e$, then there exists $\mu_{1} \in(0,1)$ such that $g(x)<0$ for $x \in\left(0, \mu_{1}\right)$ and $g(x)>0$ for $x \in\left(\mu_{1}, 1\right)$.

Proof Simple computations lead to

$$
\begin{aligned}
& g(0)=0, \quad g(1)=\frac{1}{p}-1-\frac{\pi}{2}, \\
& g^{\prime}(x)=\frac{x^{2}}{\sqrt{1-x^{2}}\left[p+(1-p) \sqrt{1-x^{2}}\right]^{2}} g_{1}(x),
\end{aligned}
$$

where

$$
g_{1}(x)=p(p-1) \sqrt{1-x^{2}}+1-2 p-p^{2} .
$$

(1) If $p=1 / 3$, then (2.11) leads to

$$
g_{1}(x)=\frac{2}{9}\left(1-\sqrt{1-x^{2}}\right)>0
$$

for $x \in(0,1)$.

Therefore, $g(x)>0$ for all $x \in(0,1)$ follows easily from (2.9), (2.10) and (2.12).

(2) If $p=1 / e$, then (2.11) leads to

$$
\begin{aligned}
& g_{1}(0)=\frac{e-3}{e}<0, \quad g_{1}(1)=\frac{e^{2}-2 e-1}{e^{2}}>0, \\
& g_{1}^{\prime}(x)=\frac{p(1-p) x}{\sqrt{1-x^{2}}}>0
\end{aligned}
$$

for all $x \in(0,1)$.

From (2.13) and (2.14) we clearly see that there exists $\mu_{0} \in(0,1)$ such that $g_{1}(x)<0$ for $x \in\left(0, \mu_{0}\right)$ and $g_{1}(x)>0$ for $x \in\left(\mu_{0}, 1\right)$. 
We divide the proof into two cases.

Case 1. $x \in\left(0, \mu_{0}\right]$. Then $g(x)<0$ for $x \in\left(0, \mu_{0}\right]$ follows easily from (2.9) and (2.10) together with $g_{1}(x)<0$ on the interval $\left(0, \mu_{0}\right)$.

Case 2. $x \in\left(\mu_{0}, 1\right)$. Then $(2.10)$ and $g_{1}(x)>0$ on the interval $\left(\mu_{0}, 1\right)$ lead to the conclusion that $g(x)$ is strictly increasing on $\left[\mu_{0}, 1\right)$. Note that

$$
g\left(\mu_{0}\right)<0, \quad g(1)=e-1-\frac{\pi}{2}>0 .
$$

From $(2.15)$ and the monotonicity of $g(x)$ on the interval $\left[\mu_{0}, 1\right)$ we clearly see that there exists $\mu_{1} \in\left(\mu_{0}, 1\right) \subset(0,1)$ such that $g(x)<0$ for $x \in\left(\mu_{0}, \mu_{1}\right)$ and $g(x)>0$ for $x \in\left(\mu_{1}, 1\right)$.

Lemma 2.3 Let $p \in(0,1 / 2)$ and

$$
h(x)=\arcsin (x)-\frac{x \sqrt{1-x^{2}}\left[1+(1-2 p)^{2} x^{2}\right]}{1-(1-2 p)^{2} x^{2}} .
$$

Then the following statements are true:

(1) If $p=1 / 2-\sqrt{3} / 6=0.2113 \ldots$, then $h(x)>0$ for all $x \in(0,1)$.

(2) If $p=1 / 2-\sqrt{1-1 / e} / 2=0.1024 \ldots$, then there exists $\sigma_{1} \in(0,1)$ such that $h(x)<0$ for $x \in\left(0, \sigma_{1}\right)$ and $h(x)>0$ for $x \in\left(\sigma_{1}, 1\right)$.

Proof Simple computations lead to

$$
\begin{aligned}
& h(0)=0, \quad h(1)=\frac{\pi}{2}, \\
& h^{\prime}(x)=-\frac{x^{2}}{\sqrt{1-x^{2}}\left[1-(1-2 p)^{2} x^{2}\right]^{2}} h_{1}(x),
\end{aligned}
$$

where

$$
\begin{aligned}
h_{1}(x)= & \left(16 p^{4}-32 p^{3}+24 p^{2}-8 p+1\right) x^{4} \\
& +\left(-16 p^{4}+32 p^{3}-32 p^{2}+16 p-3\right) x^{2}+2\left(6 p^{2}-6 p+1\right) .
\end{aligned}
$$

(1) If $p=1 / 2-\sqrt{3} / 6$, then (2.19) leads to

$$
h_{1}(x)=-\frac{4}{9} x^{2}\left(7-x^{2}\right)<0
$$

for $x \in(0,1)$.

Therefore, $h(x)>0$ for all $x \in(0,1)$ follows easily from (2.17) and (2.18) together with (2.10).

(2) If $p=1 / 2-\sqrt{1-1 / e} / 2$, then

$$
\begin{aligned}
h_{1}(0)= & 2\left(6 p^{2}-6 p+1\right)>0, \quad h_{1}(1)=-4 p(1-p)<0, \\
h_{1}^{\prime}(x)= & 4\left(16 p^{4}-32 p^{3}+24 p^{2}-8 p+1\right) x^{3} \\
& +2\left(-16 p^{4}+32 p^{3}-32 p^{2}+16 p-3\right) x .
\end{aligned}
$$


Note that

$$
\begin{aligned}
& 16 p^{4}-32 p^{3}+24 p^{2}-8 p+1=0.3995 \ldots>0 \\
& 16 p^{4}-32 p^{3}+16 p^{2}-1=-0.8646 \ldots<0
\end{aligned}
$$

It follows from (2.22)-(2.24) that

$$
\begin{aligned}
h_{1}^{\prime}(x) & <4\left(16 p^{4}-32 p^{3}+24 p^{2}-8 p+1\right) x+2\left(-16 p^{4}+32 p^{3}-32 p^{2}+16 p-3\right) x \\
& =2\left(16 p^{4}-32 p^{3}+16 p^{2}-1\right) x<0
\end{aligned}
$$

for $x \in(0,1)$.

From (2.21) and (2.25) we clearly see that there exists $\sigma_{0} \in(0,1)$ such that $h_{1}(x)>0$ for $x \in\left(0, \sigma_{0}\right)$ and $h_{1}(x)<0$ for $x \in\left(\sigma_{0}, 1\right)$.

We divide the proof into two cases.

Case 1. $x \in\left(0, \sigma_{0}\right]$. Then $h(x)<0$ for $x \in\left(0, \sigma_{0}\right]$ follows easily from (2.17) and (2.18) together with $h_{1}(x)>0$ on the interval $\left(0, \sigma_{0}\right)$.

Case 2. $x \in\left(\sigma_{0}, 1\right)$. Then $(2.18)$ and $h_{1}(x)<0$ on the interval $\left(\sigma_{0}, 1\right)$ lead to the conclusion that $h(x)$ is strictly increasing on $\left(\sigma_{0}, 1\right)$. Therefore, there exists $\sigma_{1} \in\left(\sigma_{0}, 1\right) \subset(0,1)$ such that $h(x)<0$ for $x \in\left(\sigma_{0}, \sigma_{1}\right)$ and $h(x)>0$ for $x \in\left(\sigma_{1}, 1\right)$ follows from $(2.17)$ and $h\left(\sigma_{0}\right)<0$ together with the monotonicity of $h(x)$ on the interval $\left(\sigma_{0}, 1\right)$.

Lemma 2.4 Let $p \in(0,1 / 2)$ and

$$
J(x)=\arcsin (x)-\frac{x \sqrt{1-x^{2}}}{1-(1-2 p)^{2} x^{2}}
$$

Then the following statements are true:

(1) If $p=1 / 2-\sqrt{6} / 6=0.0917 \ldots$, then $J(x)>0$ for all $x \in(0,1)$.

(2) If $p=1 / 2-\sqrt{1-1 / e^{2}} / 2=0.0350 \ldots$, then there exists $\tau_{1} \in(0,1)$ such that $J(x)<0$ for $x \in\left(0, \tau_{1}\right)$ and $h(x)>0$ for $x \in\left(\tau_{1}, 1\right)$.

Proof Simple computations lead to

$$
\begin{aligned}
& J(0)=0, \quad J(1)=\frac{\pi}{2}, \\
& J^{\prime}(x)=\frac{x^{2}}{\sqrt{1-x^{2}}\left[1-(1-2 p)^{2} x^{2}\right]^{2}} J_{1}(x),
\end{aligned}
$$

where

$$
J_{1}(x)=\left(16 p^{4}-32 p^{3}+24 p^{2}-8 p+1\right) x^{2}-\left(12 p^{2}-12 p+1\right) .
$$

(1) If $p=1 / 2-\sqrt{6} / 6$, then (2.29) leads to

$$
J_{1}(x)=\frac{4}{9} x^{2}>0
$$

for $x \in(0,1)$. 
Therefore, $J(x)>0$ for all $x \in(0,1)$ follows easily from (2.27) and (2.28) together with (2.30).

(2) If $p=1 / 2-\sqrt{1-1 / e^{2}} / 2$, then (2.29) leads to

$$
\begin{aligned}
& J_{1}(0)=-\left(12 p^{2}-12 p+1\right)<0, \quad J_{1}(1)=4 p\left(4 p^{3}-8 p^{2}+3 p+1\right)>0, \\
& J_{1}^{\prime}(x)=2\left(16 p^{4}-32 p^{3}+24 p^{2}-8 p+1\right) x>0
\end{aligned}
$$

for $x \in(0,1)$.

It follows from (2.31) and (2.32) that there exists $\tau_{0} \in(0,1)$ such that $J_{1}(x)<0$ for $x \in$ $\left(0, \tau_{0}\right)$ and $J_{1}(x)>0$ for $x \in\left(\tau_{0}, 1\right)$.

We divide the proof into two cases.

Case 1. $x \in\left(0, \tau_{0}\right]$. Then $J(x)<0$ for $x \in\left(0, \tau_{0}\right]$ follows easily from (2.27) and (2.28) together with $J_{1}(x)<0$ on the interval $\left(0, \tau_{0}\right)$.

Case 2. $x \in\left(\tau_{0}, 1\right)$. Then $(2.28)$ and $J_{1}(x)>0$ on the interval $\left(\tau_{0}, 1\right)$ lead to the conclusion that $J(x)$ is strictly increasing on $\left(\tau_{0}, 1\right)$.

Therefore, there exists $\tau_{1} \in\left(\tau_{0}, 1\right) \subset(0,1)$ such that $J(x)<0$ for $x \in\left(\tau_{0}, \tau_{1}\right)$ and $J(x)>0$ for $x \in\left(\tau_{1}, 1\right)$ follows from (2.27) and $J\left(\tau_{0}\right)<0$ together with the monotonicity of $J(x)$ on the interval $\left(\tau_{0}, 1\right)$.

\section{Main results}

Theorem 3.1 The double inequality

$$
\alpha_{1} A(a, b)+\left(1-\alpha_{1}\right) H(a, b)<X(a, b)<\beta_{1} A(a, b)+\left(1-\beta_{1}\right) H(a, b)
$$

holds for all $a, b>0$ with $a \neq b$ if and only if $\alpha_{1} \leq 1 / e=0.3678 \ldots$ and $\beta_{1} \geq 2 / 3$.

Proof Since $H(a, b), X(a, b)$ and $A(a, b)$ are symmetric and homogenous of degree one, we assume that $a>b>0$. Let $x=(a-b) /(a+b) \in(0,1)$ and $p \in(0,1)$. Then (1.1) and (1.2) lead to

$$
\begin{aligned}
& \frac{X(a, b)-H(a, b)}{A(a, b)-H(a, b)}=\frac{e^{\frac{\sqrt{1-x^{2}} \arcsin (x)}{x}-1}-\left(1-x^{2}\right)}{x^{2}} \\
& \log \frac{X(a, b)}{p A(a, b)+(1-p) H(a, b)}=\frac{\sqrt{1-x^{2}} \arcsin (x)}{x}-1-\log \left[p+(1-p)\left(1-x^{2}\right)\right] .
\end{aligned}
$$

Let

$$
F(x)=\frac{\sqrt{1-x^{2}} \arcsin (x)}{x}-1-\log \left[p+(1-p)\left(1-x^{2}\right)\right] .
$$

Then simple computations lead to

$$
\begin{aligned}
& F\left(0^{+}\right)=0, \\
& F(1)=-\log p-1, \\
& F^{\prime}(x)=\frac{1}{x^{2} \sqrt{1-x^{2}}} f(x),
\end{aligned}
$$

where $f(x)$ is defined by $(2.1)$. 
We divide the proof into two cases.

Case 1. $p=2 / 3$. Then (3.2)-(3.4) and (3.6) together with Lemma 2.1(1) lead to the conclusion that

$$
X(a, b)<\frac{2}{3} A(a, b)+\frac{1}{3} H(a, b) .
$$

Case 2. $p=1 / e$. Then (3.6) and Lemma 2.1(2) lead to the conclusion that there exists $\lambda_{1} \in(0,1)$ such that $F(x)$ is strictly increasing on $\left(0, \lambda_{1}\right]$ and strictly decreasing on $\left[\lambda_{1}, 1\right)$.

Note that (3.5) becomes

$$
F(1)=0
$$

It follows from (3.2)-(3.4) and (3.8) together with the piecewise monotonicity of $F(x)$ that

$$
X(a, b)>\frac{1}{e} A(a, b)+\left(1-\frac{1}{e}\right) H(a, b) .
$$

Note that

$$
\begin{aligned}
& \lim _{x \rightarrow 0^{+}} \frac{e^{\frac{\sqrt{1-x^{2}} \arcsin (x)}{x}-1}-\left(1-x^{2}\right)}{x^{2}}=\frac{2}{3}, \\
& \lim _{x \rightarrow 1^{-}} \frac{e^{\frac{\sqrt{1-x^{2}} \arcsin (x)}{x}-1}-\left(1-x^{2}\right)}{x^{2}}=\frac{1}{e} .
\end{aligned}
$$

Therefore, Theorem 3.1 follows from (3.7) and (3.9) in conjunction with the following statements.

- If $\alpha_{1}>2 / 3$, then equations (3.1) and (3.10) lead to the conclusion that there exists $\delta_{1} \in$ $(0,1)$ such that $X(a, b)<\alpha_{1} A(a, b)+\left(1-\alpha_{1}\right) H(a, b)$ for all $a>b>0$ with $(a-b) /(a+b) \in$ $\left(0, \delta_{1}\right)$.

-• If $\beta_{1}<1 / e$, then equations (3.1) and (3.11) lead to the conclusion that there exists $\delta_{2} \in$ $(0,1)$ such that $X(a, b)>\beta_{1} A(a, b)+\left(1-\beta_{1}\right) H(a, b)$ for all $a>b>0$ with $(a-b) /(a+b) \in$ $\left(1-\delta_{2}, 1\right)$.

Theorem 3.2 The double inequality

$$
\alpha_{2} A(a, b)+\left(1-\alpha_{2}\right) G(a, b)<X(a, b)<\beta_{2} A(a, b)+\left(1-\beta_{2}\right) G(a, b)
$$

holds for all $a, b>0$ with $a \neq b$ if and only if $\alpha_{2} \leq 1 / 3$ and $\beta_{2} \geq 1 / e=0.3678 \ldots$

Proof Since $A(a, b), G(a, b)$ and $X(a, b)$ are symmetric and homogenous of degree one, we assume that $a>b>0$. Let $x=(a-b) /(a+b) \in(0,1)$ and $p \in(0,1)$. Then (1.1) and (1.2) lead to

$$
\frac{X(a, b)-G(a, b)}{A(a, b)-G(a, b)}=\frac{e^{\frac{\sqrt{1-x^{2}} \arcsin (x)}{x}-1}-\sqrt{1-x^{2}}}{1-\sqrt{1-x^{2}}}
$$




$$
\log \frac{X(a, b)}{p A(a, b)+(1-p) G(a, b)}=\frac{\sqrt{1-x^{2}} \arcsin (x)}{x}-1-\log \left[p+(1-p) \sqrt{1-x^{2}}\right] .
$$

Let

$$
G(x)=\frac{\sqrt{1-x^{2}} \arcsin (x)}{x}-1-\log \left[p+(1-p) \sqrt{1-x^{2}}\right] .
$$

Then simple computations lead to

$$
\begin{aligned}
& G\left(0^{+}\right)=0, \\
& G(1)=-\log p-1, \\
& G^{\prime}(x)=\frac{1}{x^{2} \sqrt{1-x^{2}}} g(x),
\end{aligned}
$$

where $g(x)$ is defined by $(2.8)$.

We divide the proof into two cases.

Case 1. $p=1 / 3$. Then (3.13)-(3.15) and (3.17) together with Lemma 2.2(1) lead to the conclusion that

$$
X(a, b)>\frac{1}{3} A(a, b)+\frac{2}{3} G(a, b) .
$$

Case 2. $p=1 / e$. Then from Lemma 2.2(2) and (3.17) we know that there exists $\mu_{1} \in(0,1)$ such that $G(x)$ is strictly decreasing on $\left(0, \mu_{1}\right]$ and strictly increasing on $\left[\mu_{1}, 1\right)$. Note that (3.16) becomes

$$
G(1)=0
$$

It follows from (3.13)-(3.15) and (3.19) together with the piecewise monotonicity of $G(x)$ that

$$
X(a, b)<\frac{1}{e} A(a, b)+\left(1-\frac{1}{e}\right) G(a, b) .
$$

Note that

$$
\begin{aligned}
& \lim _{x \rightarrow 0^{+}} \frac{e^{\frac{\sqrt{1-x^{2}} \arcsin (x)}{x}-1}-\sqrt{1-x^{2}}}{1-\sqrt{1-x^{2}}}=\frac{1}{3}, \\
& \lim _{x \rightarrow 1^{-}} \frac{e^{\frac{\sqrt{1-x^{2}} \arcsin (x)}{x}-1}-\sqrt{1-x^{2}}}{1-\sqrt{1-x^{2}}}=\frac{1}{e} .
\end{aligned}
$$

Therefore, Theorem 3.2 follows easily from (3.12) and (3.18) together with (3.20)(3.22).

Theorem 3.3 Let $\alpha_{3}, \beta_{3} \in(0,1 / 2)$. Then the double inequality

$$
H\left[\alpha_{3} a+\left(1-\alpha_{3}\right) b, \alpha_{3} b+\left(1-\alpha_{3}\right) a\right]<X(a, b)<H\left[\beta_{3} a+\left(1-\beta_{3}\right) b, \beta_{3} b+\left(1-\beta_{3}\right) a\right]
$$


holds for all $a, b>0$ with $a \neq b$ if and only if $\alpha_{3} \leq 1 / 2-\sqrt{1-1 / e} / 2=0.1024 \ldots$ and $\beta_{3} \geq$ $1 / 2-\sqrt{3} / 6=0.2113 \ldots$

Proof Since $H(a, b)$ and $X(a, b)$ are symmetric and homogenous of degree one, we assume that $a>b>0$. Let $x=(a-b) /(a+b) \in(0,1)$ and $p \in(0,1 / 2)$. Then (1.1) and (1.2) lead to

$$
\log \frac{H[p a+(1-p) b, p b+(1-p) a]}{X(a, b)}=\log \left[1-(1-2 p)^{2} x^{2}\right]-\frac{\sqrt{1-x^{2}} \arcsin (x)}{x}+1
$$

Let

$$
H(x)=\log \left[1-(1-2 p)^{2} x^{2}\right]-\frac{\sqrt{1-x^{2}} \arcsin (x)}{x}+1
$$

Then simple computations lead to

$$
\begin{aligned}
& H\left(0^{+}\right)=0, \\
& H(1)=1+2 \log 2+\log \left(p-p^{2}\right), \\
& H^{\prime}(x)=\frac{1}{x^{2} \sqrt{1-x^{2}}} h(x),
\end{aligned}
$$

where $h(x)$ is defined by $(2.16)$.

We divide the proof into four cases.

Case 1. $p=1 / 2-\sqrt{3} / 6$. Then (3.23)-(3.25) and (3.27) together with Lemma 2.3(1) lead to

$$
X(a, b)<H\left[\left(\frac{1}{2}-\frac{\sqrt{3}}{6}\right) a+\left(\frac{1}{2}+\frac{\sqrt{3}}{6}\right) b,\left(\frac{1}{2}-\frac{\sqrt{3}}{6}\right) b+\left(\frac{1}{2}+\frac{\sqrt{3}}{6}\right) a\right] .
$$

Case 2. $0<p<1 / 2-\sqrt{3} / 6$. Let $q=(1-2 p)^{2}$ and $x \rightarrow 0^{+}$, then $1 / 3<q<1$ and power series expansion leads to

$$
H(x)=-\left(q-\frac{1}{3}\right) x^{2}+o\left(x^{2}\right)
$$

Equations (3.23), (3.24) and (3.28) lead to the conclusion that there exists $0<\delta<1$ such that

$$
X(a, b)>H[p a+(1-p) b, p b+(1-p) a]
$$

for all $a>b>0$ with $(a-b) /(a+b) \in(0, \delta)$.

Case 3. $p=1 / 2-\sqrt{1-1 / e} / 2$. Then (3.27) and Lemma 2.3(2) lead to the conclusion that there exists $\sigma_{1} \in(0,1)$ such that $H(x)$ is strictly decreasing on $\left(0, \sigma_{1}\right]$ and strictly increasing on $\left[\sigma_{1}, 1\right)$.

Note that (3.26) becomes

$$
H(1)=0 .
$$


Therefore,

$$
X(a, b)>H\left[\left(\frac{1}{2}-\frac{\sqrt{1-\frac{1}{e}}}{2}\right) a+\left(\frac{1}{2}+\frac{\sqrt{1-\frac{1}{e}}}{2}\right) b,\left(\frac{1}{2}-\frac{\sqrt{1-\frac{1}{e}}}{2}\right) b+\left(\frac{1}{2}+\frac{\sqrt{1-\frac{1}{e}}}{2}\right) a\right]
$$

follows from (3.23)-(3.25) and (3.30) together with the piecewise monotonicity of $H(x)$.

Case $4.1 / 2-\sqrt{1-1 / e} / 2<p<1 / 2$. Then (3.26) leads to

$$
H(1)>0 \text {. }
$$

Equations (3.23) and (3.24) together with inequality (3.31) imply that there exists $0<$ $\delta^{\prime}<1$ such that

$$
X(a, b)<H[p a+(1-p) b, p b+(1-p) a]
$$

for $a>b>0$ with $(a-b)(a+b) \in\left(1-\delta^{\prime}, 1\right)$.

Theorem 3.4 Let $\alpha_{4}, \beta_{4} \in(0,1 / 2)$. Then the double inequality

$$
G\left[\alpha_{4} a+\left(1-\alpha_{4}\right) b, \alpha_{4} b+\left(1-\alpha_{4}\right) a\right]<X(a, b)<G\left[\beta_{4} a+\left(1-\beta_{4}\right) b, \beta_{4} b+\left(1-\beta_{4}\right) a\right]
$$

holds for all $a, b>0$ with $a \neq b$ if and only if $\alpha_{4} \leq 1 / 2-\sqrt{1-1 / e^{2}} / 2=0.0350 \ldots$ and $\beta_{4} \geq$ $1 / 2-\sqrt{6} / 6=0.0917 \ldots$

Proof Since $G(a, b)$ and $X(a, b)$ are symmetric and homogenous of degree one, we assume that $a>b>0$. Let $x=(a-b) /(a+b) \in(0,1)$ and $p \in(0,1 / 2)$. Then (1.1) and (1.2) lead to

$$
\begin{aligned}
\log & \frac{G[p a+(1-p) b, p b+(1-p) a]}{X(a, b)} \\
& =\frac{1}{2} \log \left[1-(1-2 p)^{2} x^{2}\right]-\frac{\sqrt{1-x^{2}} \arcsin (x)}{x}+1 .
\end{aligned}
$$

Let

$$
K(x)=\frac{1}{2} \log \left[1-(1-2 p)^{2} x^{2}\right]-\frac{\sqrt{1-x^{2}} \arcsin (x)}{x}+1 .
$$

Then simple computations lead to

$$
\begin{aligned}
& K\left(0^{+}\right)=0, \\
& K(1)=1+\log 2+\frac{1}{2} \log \left(p-p^{2}\right), \\
& K^{\prime}(x)=\frac{1}{x^{2} \sqrt{1-x^{2}}} J(x),
\end{aligned}
$$

where $J(x)$ is defined by $(2.26)$.

We divide the proof into four cases. 
Case 1. $p=p_{0}=1 / 2-\sqrt{6} / 6$. Then

$$
X(a, b)<G\left[p_{0} a+\left(1-p_{0}\right) b, p_{0} b+\left(1-p_{0}\right) a\right]
$$

follows from (3.32)-(3.34) and (3.36) together with Lemma 2.4(1).

Case 2. $0<p<1 / 2-\sqrt{6} / 6$. Let $q=(1-2 p)^{2}$ and $x \rightarrow 0^{+}$, then $2 / 3<q<1$ and power series expansion leads to

$$
K(x)=-\frac{1}{2}\left(q-\frac{2}{3}\right) x^{2}+o\left(x^{2}\right) .
$$

From (3.32), (3.33) and (3.37) we clearly see that there exists $0<\delta<1$ such that

$$
X(a, b)>G[p a+(1-p) b, p b+(1-p) a]
$$

for $a>b>0$ with $(a-b) /(a+b) \in(0, \delta)$.

Case 3. $p=p_{1}=1 / 2-\sqrt{1-1 / e^{2}} / 2$. Then (3.36) and Lemma 2.4(2) lead to the conclusion that there exists $\tau_{1} \in(0,1)$ such that $K(x)$ is strictly decreasing on $\left(0, \tau_{1}\right]$ and strictly increasing on $\left[\tau_{1}, 1\right)$.

Note that (3.35) becomes

$$
K(1)=0 .
$$

Therefore,

$$
X(a, b)>G\left[p_{1} a+\left(1-p_{1}\right) b, p_{1} b+\left(1-p_{1}\right) a\right]
$$

follows from (3.32)-(3.34) and (3.38) together with the piecewise monotonicity of $K(x)$.

Case $4.1 / 2-\sqrt{1-1 / e^{2}} / 2<p<1 / 2$. Then (3.35) leads to

$$
K(1)>0 .
$$

Equations (3.32) and (3.33) together with inequality (3.39) imply that there exists $0<$ $\delta^{\prime}<1$ such that

$$
X(a, b)<G[p a+(1-p) b, p b+(1-p) a]
$$

for $a>b>0$ with $(a-b) /(a+b) \in\left(1-\delta^{\prime}, 1\right)$.

Competing interests

The authors declare that they have no competing interests.

Authors' contributions

All authors contributed equally to the writing of this paper. All authors read and approved the final manuscript.

\section{Author details}

'School of Distance Education, Huzhou Broadcast and TV University, Huzhou, 313000, China. ${ }^{2}$ School of Mathematics and Computation Sciences, Hunan City University, Yiyang, 413000, China. 


\section{Acknowledgements}

The research was supported by the Natural Science Foundation of China under Grants 61374086, 11171307 and

11401191, and the Natural Science Foundation of Zhejiang Province under Grant LY13A010004.

Received: 23 February 2015 Accepted: 23 June 2015 Published online: 09 July 2015

\section{References}

1. Sándor, J: Two sharp inequalities for trigonometric and hyperbolic functions. Math. Inequal. Appl. 15(2), 409-413 (2012)

2. Sándor, J: On two new means of two variables. Notes Number Theory Discrete Math. 20(1), 1-9 (2014)

3. Yang, Z-H, Wu, L-M, Chu, Y-M: Sharp power mean bounds for Sándor mean. Abstr. Appl. Anal. 2014, Article ID 172867 (2014)

4. Zhou, S-S, Qian, W-M, Chu, Y-M, Zhang, X-H: Sharp power-type Heronian mean bounds for the Sándor and Yang means. J. Inequal. Appl. 2015, Article ID 159 (2015)

Submit your manuscript to a SpringerOpen ${ }^{\odot}$ journal and benefit from:

- Convenient online submission

- Rigorous peer review

- Immediate publication on acceptance

- Open access: articles freely available online

- High visibility within the field

- Retaining the copyright to your article 